\title{
Análisis por el método de elementos finitos del comportamiento de las pastillas de freno ABS con base de acero y zinc al discretizar el elemento continuo al utilizar "software" CAE
}

\section{(Analysis by the finite element method of the behavior of the ABS brake pads with materials based on steel and zinc discretizing the continuous element using CAE software)}

\author{
Alexy Fabian Vinueza Lozada ${ }^{1}$, Nelson Ramiro Gutiérrez Suquillo²
}

\begin{abstract}
Resumen:
En el presente trabajo se realizó el estudio del comportamiento de las pastillas de freno de un sistema ABS utilizando el método de análisis por elementos finitos, donde, una de las acciones más críticas es la de realizar un mallado de calidad, para mejorar la convergencia de resultados optimizando la carga computacional; esto posibilita que el problema pueda ser resuelto en un menor tiempo. El uso de herramientas de software para realizar este tipo de estudio tiene la ventaja que en el posprocesamiento se puede escoger el tipo de parámetros que se quieren estudiar, en este caso se analizaron variables mecánicas y térmicas que permitieron caracterizar el comportamiento de los materiales de las pastillas. El estudio se efectuó para dos materiales: Trimat MN 1081 que está compuesto de acero y filamentos no ferrosos y Trimat GZC compuesto de fibras de zinc, de esta manera se obtuvieron características importantes de estos materiales, como son el esfuerzo, la deformación, penetración, flujo de calor y energía. A base del método desarrollado y a los resultados obtenidos, se realizará un estudio con materiales alternativos, lo cual permitirá determinar la viabilidad de usarlos en busca de obtener resultados óptimos tanto en características mecánicas como en costos.
\end{abstract}

Palabras claves: mallado; simulación computacional; desgaste; flujo de calor; criterio de convergencia.

\begin{abstract}
:
In this paper, the study of the behavior of the brake pads of an ABS system was carried out using the finite element analysis method, where one of the most critical actions is to perform quality meshing, so that this allows improving the convergence of results by optimizing the computational load, which enables the problem to be solved in less time. The use of software tools to carry out this type of study has the advantage that in the post-processing the type of parameters that can be studied can be chosen, in this case, mechanical and thermal variables that allowed to characterize the behavior of the materials were analyzed. The study was carried out for two materials: Trimat MN1081 which is composed of steel and non-ferrous filaments and Trimat GZC composed of zinc fibers, in this way important characteristics of these materials were obtained, such as stress, deformation, penetration, the flow heat and energy. Based on the method developed and the results obtained, a study will be conducted with alternative materials, which will allow determining the feasibility of using these materials in search of optimal results in both mechanical characteristics and costs.
\end{abstract}

Keywords: mesh; computational simulation; wear; heat flux; convergence.

\footnotetext{
${ }^{1}$ Universidad Tecnológica Equinoccial, Quito, Ecuador ( \{alexy.vinueza, nelson.gutierrez\} @ute.edu.ec ).
} 


\section{Introducción}

El análisis por el método de elementos finitos (FEA) es la solución a muchos problemas de ingeniería, caracterizado por modelos matemáticos más o menos complejos en los cuales intervienen un conjunto de parámetros para predecir fallos en todo sistema físico continuo con un material específico determinado. (Naula I., Albuja, G.; Carrillo, R; Izurieta, C., 2016).

Este tipo de análisis constituye una alternativa de mejora al método tradicional de diseño y desarrollo usado en ingeniería denominado de "prueba y error", en el cual se tienen costos y tiempos de manufactura para desarrollar un prototipo, el cual genera limitaciones para estudiar la totalidad del sistema físico y solo se pueden verificar determinados puntos. (Benito Muñoz J. J., Álvarez Cabal R., Ureña Prieto F., Salete Casino E., Aranda Ortega E., 2014)

Para soluciones de ingeniería, estos se centran en la eficiencia de la combustión, la interferencia electrónica, la fiabilidad estructural, las interfaces hombre-máquina o la robustez térmica, etc. (ANSYS, Ansys advantage-magazine, 2017).

Este método ayuda a investigar los efectos físicos, innovando y optimizando el diseño global del producto y sus aplicaciones pueden ser: para rendimiento de antenas, refrigeración electrónica, electromagnetismo de baja frecuencia, electrónica de potencia, software incrustado, sistemas multi-físicos, estructurales de impacto, esfuerzos, térmicos, vibraciones, sistemas de prototipos virtuales etc. (ANSYS, Ansys advantage-magazine, 2017).

Se han realizado trabajos por este método como el análisis de un disco de freno con sistema de mordaza (Venegas, 2012). Automation Structural Analysis Based on the VB and ANSYS (Feng Liu, Xiankuan Qi and Mingqing Sun, 2008). Dynamics Analysis of the MRF Rectangular Sandwich Plate Based on ANSYS (ZhengXin Zhang; FangLin Huang, 2013). FEA of In-Plane Shear Stresses of a Preloaded Sandwich Plate with a Viscoelastic Core: Application to the Disk Brake System (Maher Bouazizi; Tarek Lazghab; Mohamed Soula, 2013).

Mediante el análisis y simulación con herramientas de Ingeniería Asistida por Computador - CAE, se pueden validar los Diseños Modelados por Computador - CAD (García, A., Castillo, F., 2007).

El análisis dinámico modal es una técnica utilizada para determinar las características del movimiento vibratorio de una estructura, mediante este tipo de estudio se pueden encontrar las siguientes características:

- Frecuencias naturales, estructuras con vibración natural.

- Factores de participación, cantidad de masa en la estructura donde cada modo toma una dirección.

- Modos o formas de vibrar, estructura vibra de acuerdo con la frecuencia. Una estructura vibra de acuerdo con los grados de libertad que posee.

El análisis modal es el más básico de todos, donde el diseñador determina las frecuencias naturales para evitar vibración de frecuencias o efectos vibratorios en resonancia que ocasionan fallas en el diseño (ANSYS, Ansys advantage-magazine, 2012).

En este caso en particular, se analiza el comportamiento termo-mecánico de las pastillas con materiales convencionales y alternativos de un sistema de frenos ABS. La pastilla presiona el material de fricción contra la cara o las caras del disco-rotativo. El trabajo desarrollado es complementario al artículo realizado por (Vinueza A., Gutierrez N., 2017).

La Figura 1 muestra la geometría de un área de contacto de freno frotador anular (R. Budynas, N. Keith, 2011). 

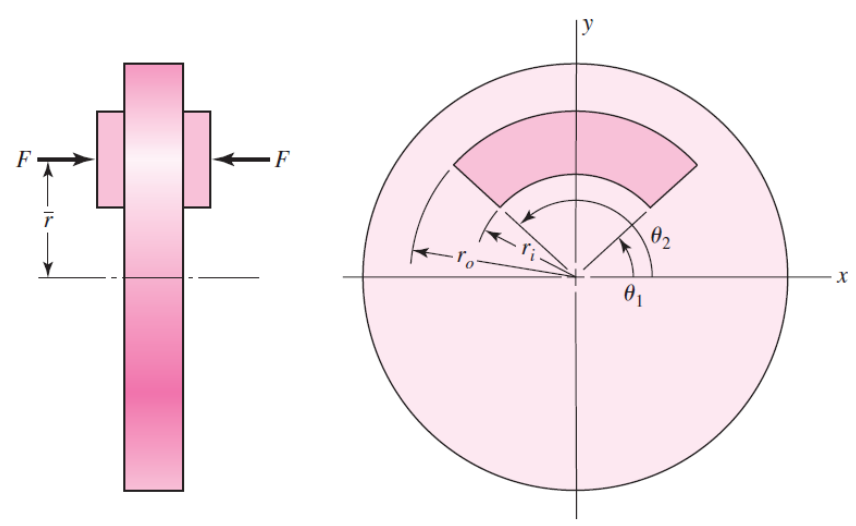

Figura 1. Geometría de área de contacto (R. Budynas, N. Keith, 2011)

La fuerza de rozamiento o fricción se origina básicamente entre la superficie rugosa de los cuerpos en contacto. A pesar que a simple vista tengan apariencia lisa como se puede observar en la Figura 2 (R. Budynas, N. Keith, 2011) (Norton, 2010).

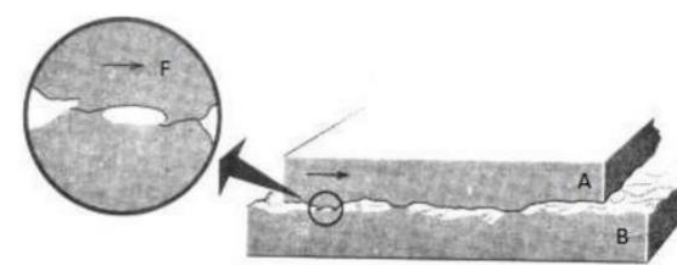

Figura 2. Rozamiento entre dos superficies. (Falasca, 2016)

Lo ideal de un material de fricción es mantener constante el coeficiente durante todo el tiempo de trabajo y con todas las variables que influyen sobre las pastillas, como temperatura, desgaste, etc. Lo complicado es mantener estable este valor debido a los cambios en el material y se muestra en la Figura 3.

El fading es uno de los fenómenos más peligrosos que se pueden dar en un sistema de freno, y es la pérdida de eficacia de la frenada en caliente (Falasca, 2016) ( Cornejo C., 2010).

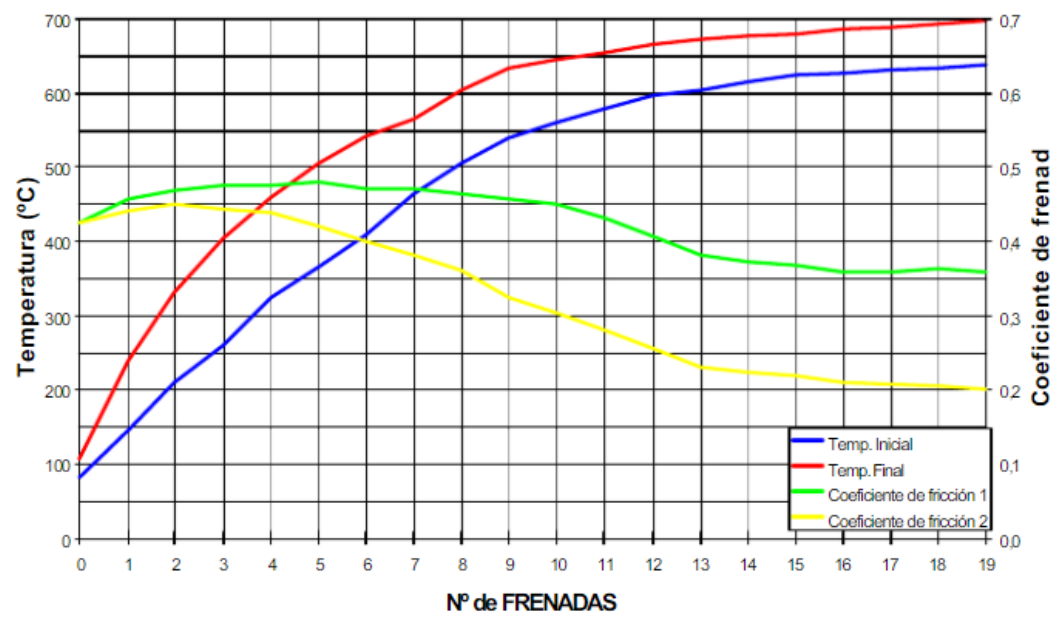

Figura 3. Gráfica obtenida de las etapas de la prueba de Fading. (Falasca, 2016)

A partir de un diseño conceptual y matemático se puede realizar un dibujo CAD adecuado y así poder efectuar FEA mediante software CAE, a partir de condiciones de borde y de frontera para las decisiones preliminares del tipo de análisis, modelo 
matemático, tipo de elemento, luego definir un preprocesamiento con la geometría, asignando un material, estableciendo un mallado de calidad (ANSYS, Ansys advantagemagazine, 2012).

El mallado es una parte fundamental para obtener una solución óptima para una mayor convergencia y finalmente la revisión con la validación de los resultados en el posprocesamiento (ANSYS, Ansys advantage-magazine, 2017).

\section{Metodología}

El modelo en $\mathrm{V}$, relaciona actividades probando el análisis y diseño como se muestra en la Figura 4. La unión mediante líneas discontinuas entre fases de la izquierda con la derecha representa doble flujo de información, por un lado se definen pruebas correspondientes, mientras que por el otro, sirve para encontrar fallos en el desarrollo, y retroalimentar hasta cumplir el objetivo propuesto (Forsberg, K., Mooz, H., Cotterman, H, 2005).

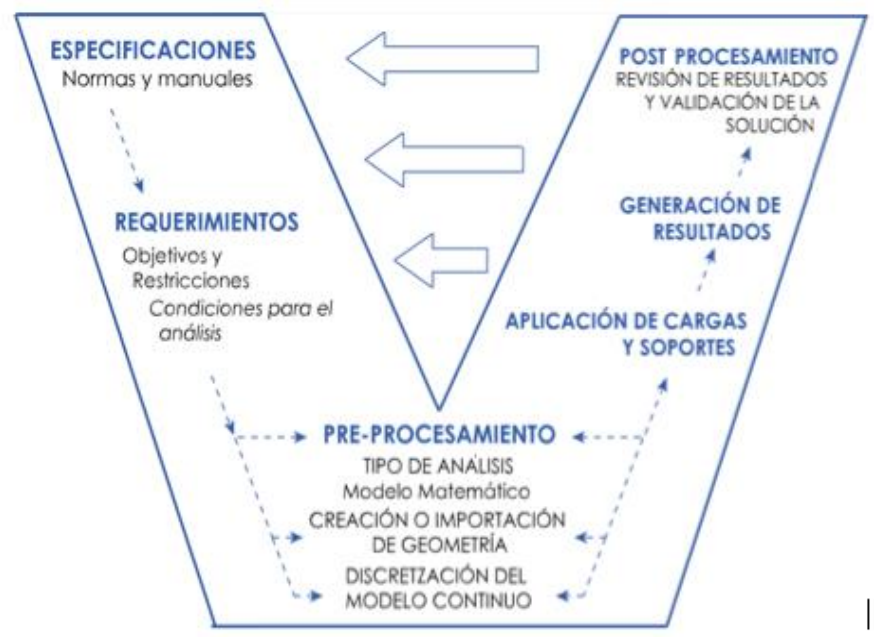

Figura 4. Modelo en V.

\subsection{Especificaciones}

Los valores establecidos de acuerdo con la prueba "A" de presión y área de contacto real, al retirar ranuras, chaflanes y redondeos mostrados en la Tabla 1.

Tabla 1. Valores establecidos para la prueba " $A$ "

\begin{tabular}{|l|l|l|l|}
\hline Ensamblaje & $\begin{array}{l}\text { Máxima presión } \\
\text { de la superficie } \\
\text { en contacto } \\
\text { [Mpa] }\end{array}$ & $\begin{array}{c}\text { Aplicación de } \\
\text { presión en } \\
\text { función del } \\
\text { tiempo [Mpa/s] }\end{array}$ & $\begin{array}{c}\text { Valor de } \\
\text { cabezal Control } \\
\text { de velocidad } \\
\text { [mm/min] }\end{array}$ \\
\hline Disco de freno & $8^{\mathrm{a}}$ & $4.0 \pm 0.5$ & 15 \\
\hline Freno de tambor & 5 & $2.5 \pm 0.5$ & 10 \\
\hline $\begin{array}{l}\text { Protección de } \\
\text { freno de tambor }\end{array}$ & 3 & $1.5 \pm 0.5$ & 6 \\
\hline $\begin{array}{l}\text { a valor establecido para pastillas más largas, 5MPa es una alternativa de } \\
\text { presión usada. }\end{array}$
\end{tabular}

Fuente: (ISO6310, 2009) 


\subsection{Requerimientos}

Las variables, rangos de operación y errores máximos recomendados están en la Tabla 2.

Tabla 2. Variables, rangos de operación y errores máximos recomendados

\begin{tabular}{|l|l|l|}
\hline Variable & \multicolumn{1}{|c|}{$\begin{array}{c}\text { Típico rango de } \\
\text { operación }\end{array}$} & \multicolumn{1}{|c|}{$\begin{array}{c}\text { Error máximo } \\
\text { recomendado }\end{array}$} \\
\hline Velocidad longitudinal inicial $^{\mathrm{a}}$ & $102 \mathrm{~km} / \mathrm{h}-98 \mathrm{~km} / \mathrm{h}$ & $\pm 0.5 \mathrm{~km} / \mathrm{h}$ \\
\hline Velocidad longitudinal $^{\mathrm{b}}$ & $93 \mathrm{~km} / \mathrm{h}-5 \mathrm{~km} / \mathrm{h}$ & $\pm 0.5 \mathrm{~km} / \mathrm{h}$ \\
\hline Distancia longitudinal & $100 \mathrm{~m}$ & $\begin{array}{l} \pm 1 \%(\leq 50 \mathrm{~m}) \\
\pm 0.5 \mathrm{~m}(>50 \mathrm{~m})\end{array}$ \\
\hline Fuerza de disparo de freno & $\leq 10 \mathrm{~N}$ (punto de disparo) & $\pm 5 \mathrm{~N}$ \\
\hline Fuerza de aplicación de freno & $\begin{array}{l}\mathrm{N}-1000 \mathrm{~N} \mathrm{(Max.} \\
1500 \mathrm{~N})\end{array}$ & $\pm 2 \%$ \\
\hline $\begin{array}{l}\text { a Determinado en un intervalo promedio 0.2s }-0 \text { s antes del contacto con el pedal } \\
\text { del freno. } \\
\text { b Desviaciones de la velocidad medida son normales en aplicación total de freno. }\end{array}$ \\
\hline
\end{tabular}

Fuente: (ISO21994, 2007)

Las características se obtienen de la ficha técnica del auto marca AVEO SEDAN 1.6 y sus parámetros a utilizar están en la Tabla 3.

Tabla 3. Ficha técnica AVEO SEDAN 1.6

\begin{tabular}{|l|l|l|l|}
\hline Motor & & Frenos ABS & SI \\
\hline Tipo & DOHC & Freno delantero & Disco ventilado \\
\hline Desplazamiento & 1598 & Freno trasero & Tambor \\
\hline $\begin{array}{l}\text { Potencia(HP@ } \\
\text { rpm) }\end{array}$ & $103 @ 5800$ & $\begin{array}{l}\text { Llantas } \\
\text { frontales }\end{array}$ & $185 / 60$ R14 \\
\hline $\begin{array}{l}\text { Torque(N.m@ } \\
\text { rpm) }\end{array}$ & $114.15 @ 3600$ & $\begin{array}{l}\text { Llantas } \\
\text { Traseras }\end{array}$ & $185 / 60$ R14 \\
\hline
\end{tabular}

El material y la composición de las pastillas de freno (Trimat MN1081 y Trimat GZC) para el análisis vienen dadas en el product data sheet de la empresa "TRIMAT friction solutions" que tiene un registro de calidad certificada BS EN ISO 9001:2008 (TRIMAT friction, Trimat friction solutions, 2012).

\subsection{Preprocesamiento}

Se analiza el fenómeno del frenado, observando que las principales variantes son: calor generado por la fricción, vibración y esfuerzos. Al saber que los módulos de ANSYS permiten realizar este tipo de análisis y que trabajan como medios independientes y/o acoplados, para este sistema se analiza con Static-Estructural, Steady-State Thermal y Modal; se muestre un esquema de proyecto en la Figura 5. 


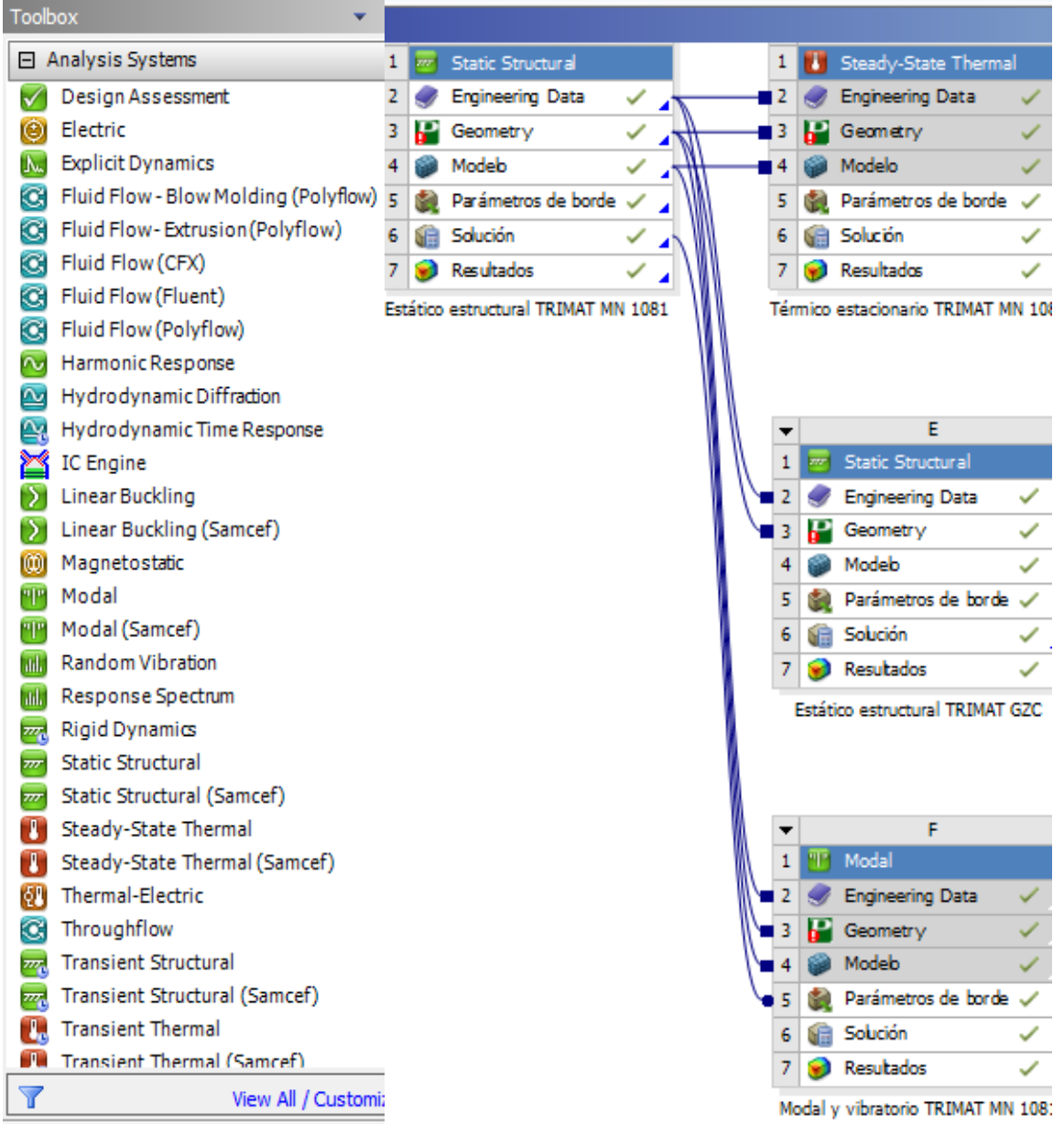

Figura 5. Esquema de proyecto mediante el uso de un software CAE por elementos finitos

Una parte del modelo matemático que trabaja el software viene descrito a continuación.

La fuerza de accionamiento $F$, la presión local de contacto $p$, el par de torsión de fricción Tfr, los ángulos inicial y final de la pastilla $\theta 1$ y $\theta 2$ están dados por: (R. Budynas, N. Keith, 2011) ( Cornejo C., 2010).

$$
\begin{gathered}
F=\int_{\theta_{1}}^{\theta_{2}} \int_{r_{i}}^{r_{o}} p r d r d \theta=\left(\theta_{2}-\theta_{1}\right) \int_{r_{i}}^{r_{o}} p r d r \\
\mathrm{~T}_{\mathrm{fr}}=\int_{\theta_{1}}^{\theta_{2}} \int_{\mathrm{r}_{\mathrm{i}}}^{\mathrm{r}_{\mathrm{o}}} \mathrm{fpr}^{2} \mathrm{drd} \theta=\left(\theta_{2}-\theta_{1}\right) \mathrm{f} \int_{\mathrm{r}_{\mathrm{i}}}^{\mathrm{r}_{\mathrm{o}}} \mathrm{pr}^{2} \mathrm{dr}
\end{gathered}
$$

El radio equivalente $r_{e}$ se determina mediante:

$$
r_{e}=\frac{T}{f F}=\frac{\int_{r_{i}}^{r_{o}} p r^{2} d r}{\int_{r_{i}}^{r_{o}} p r d r}
$$

Coordenada de ubicación $r$ se calcula tomando momentos respecto al eje $x$ :

$$
\begin{gathered}
M_{x}=F r=\int_{\theta_{1}}^{\theta_{2}} \int_{r_{i}}^{r_{o}} \operatorname{pr}(r \operatorname{sen} \theta) d r d \theta \\
M_{x}=F r=\left(\cos \theta_{1}-\cos \theta_{2}\right) \int_{r_{i}}^{r_{o}} p r^{2} d r
\end{gathered}
$$




$$
r=\frac{M_{x}}{F}=\frac{\left(\cos \theta_{1}-\cos \theta_{2}\right)}{\theta_{2}-\theta_{1}} * r_{e}
$$

Para el desgaste uniforme se utiliza la máxima presión permisible " $p_{a}$ " que se presenta en el radio interno " $r_{i}$ " dando a conocer que $p=p_{a} * r_{i} / r$ convirtiendo la ecuación (1) en:

$$
F_{p}=\left(\theta_{2}-\theta_{1}\right) p_{a} * r_{i}\left(r_{o}-r_{i}\right)
$$

La ecuación (2) en:

$$
\begin{gathered}
T_{f r}=\left(\theta_{2}-\theta_{1}\right) * f * p_{a} * r_{i} \int_{r_{i}}^{r_{o}} r d r \\
T_{f r}=\frac{1}{2}\left(\theta_{2}-\theta_{1}\right) f * p_{a} * r_{i}\left(r^{2} o-r^{2} i\right)
\end{gathered}
$$

La ecuación (3) en:

$$
r_{e}=\frac{p_{a} r_{i}\left[\int_{r_{i}}^{r_{o}} r d r\right]}{p_{a} r_{i}\left[\int_{r_{i}}^{r_{o}} d r\right]}=\frac{r_{o}+r_{i}}{2}
$$

La ecuación (5) en:

$$
r=\frac{\left(\cos \theta_{1}-\cos \theta_{2}\right)}{\theta_{2}-\theta_{1}}\left(\frac{r_{o}+r_{i}}{2}\right)
$$

Se considera a la fricción del disco de frenos con el área diferencial de contacto por la pastilla: (R. Budynas, N. Keith, 2011) (Norton, 2010) (Robert, 2002).

$$
d A=r * d \theta * d r
$$

Y la fuerza normal sobre ella:

$$
d F=p * d A
$$

dónde:

$d A=$ Área diferencial de la pastilla en contacto con el disco.

$d \theta=$ Ángulo diferencial de contacto de la pastilla.

$d r=$ Radio diferencial de contacto de la pastilla .

$r_{o}=$ Radio exterior del disco.

$r_{i}=$ Radio interior del disco.

$p=$ Presión hidráulica.

La energía absorbida por el freno depende del tipo de movimiento que tenga el cuerpo, se considera al vehículo como una masa a una velocidad $v 1$, se reduce por el efecto causado por el freno a una v2. (Venegas, 2012) (S. Chand, R. Khurmi, J. Gupta, 2005).

$$
\Delta K E=\frac{1}{2} m\left[v_{x}(t-\Delta t)^{2}-v_{x}(t)^{2}\right]
$$

La variación de tiempo $\Delta t$ hasta que el vehículo se detenga $v_{x}(t)^{2}=0$, el cambio de energía es: 


$$
\Delta K E=\frac{1}{2} m\left[v_{x}(t-\Delta t)^{2}\right]
$$

Se toma como uniforme la desaceleración, y el cambio de velocidad es una función lineal de tiempo, es decir:

$$
v_{x}(t)=v_{x}\left(1-\frac{t}{t_{f}}\right)
$$

Con la disminución de la velocidad se obtiene: (T. Brown, H. Rothbart, 2006).

$$
\begin{gathered}
v_{x 1}=v_{x}\left(1-\frac{t-\Delta t}{t_{f}}\right) \\
v_{x 2}=v_{x}\left(1-\frac{t}{t_{f}}\right)
\end{gathered}
$$

Al final, la variación de la energía cinética en el disco y pastillas de freno: (T. Brown, H. Rothbart, 2006).

$$
\Delta K E=\frac{1}{2 * 12.96} m\left[v_{x 1}^{2} v_{x 2}^{2}\right]
$$

dónde:

$\Delta K E$ = Cambio de la energía cinética de traslación, [J].

$\Delta t=$ Incremento de tiempo de reducción de velocidad, [s].

$t_{f}=$ Tiempo de frenado, [s].

$v_{x}(t)=$ Velocidad del automóvil en función del tiempo, $[\mathrm{Km} / \mathrm{h}]$.

$v_{x 1}=$ Velocidad inicial $[\mathrm{Km} / \mathrm{h}] ; v_{x 2}=$ Velocidad final, $[\mathrm{Km} / \mathrm{h}]$.

$r_{n}=$ Radio del neumático, $[\mathrm{mm}]$.

El calor disipado durante el frenado es:

$$
q=\frac{\eta * i_{a}}{4186.8} * \frac{\Delta K E}{\Delta t}
$$
manera:

Factor de corrección por masas rodantes también se encuentra de la siguiente

$$
i_{a}=\frac{A_{i}}{A}
$$

El calor disipado en el disco se calcula: (S. Chand, R. Khurmi, J. Gupta, 2005).

$$
q_{a}=227.8 \mu * p_{\max } * v_{x 1}
$$

dónde:

$q=$ Calor disipado durante el frenado, [Kcal/s].

$q_{a}=$ Calor producido en el disco de freno, $\left[\mathrm{KW} / \mathrm{m}^{2}\right]$.

$\eta=$ Porcentaje de distribución de fuerza para el disco.

$A_{i}=$ Incidencia de área de la pastilla en movimiento.

$A=$ Área de la pastilla de freno.

$i_{a}=$ Factor de corrección para masas rodantes, para autos $1.05 \leq i_{a} \leq 1.5$

Para la temperatura de frenado: (Venegas, 2012).

$$
T=\frac{T_{f r} * W * t_{f} * m_{d^{*}} * c_{d} * T_{o} * h * A_{c} * T_{a m b}}{m_{d^{*} c_{d}}+h * A_{c}}
$$


dónde:

$T=$ Temperatura del disco de freno, $\left[{ }^{\circ} \mathrm{C}\right]$.

$T_{o}=$ Temperatura inicial en el disco de freno, $\left[{ }^{\circ} \mathrm{C}\right]$.

$h=$ Coeficiente de convección del disco de freno, $\left[\mathrm{W} / \mathrm{mm}^{2} .{ }^{\circ} \mathrm{C}\right]$.

$A_{c}=$ Área de convección del disco de freno (área de la pastilla), [ $\left.\mathrm{mm}^{2}\right]$.

$c_{d}=$ Capacidad de calor específico del disco de freno, $\left[\mathrm{J} / \mathrm{kg}^{\circ} . \mathrm{C}\right]$.

$Q_{c}=$ Calor por convección, [J].

$W_{f r}=$ Trabajo por fricción, [J].

Para la importación de la geometría, primero se han modelado en CAD sus partes y su ensamble, donde el sistema de coordenadas está en el centroide del disco. Para el criterio de mallado y su generación dividiendo a la geometría continua en muchos elementos discretos (Venegas, 2012).

Debido a que la convergencia de la solución depende de la calidad de la malla, es necesario hacer un buen uso de las herramientas de mallado y evitar problemas de peso computacional (Venegas, 2012).

Se utiliza un mallado estructural hexaédrico para las pastillas y tetraédrico para el disco debido a su geometría (Díaz, 2000).

En la Figura 6 se muestra el mallado de la pastilla utilizando un Sweep Method con un Body Sizing y así mejorar de manera estructural, analizando con el criterio de calidad en oblicuidad (Skewness) para garantizar la convergencia y estar en un parámetro de 00.25 como excelente y de 0.25-0.5 como bueno (Díaz, 2000).

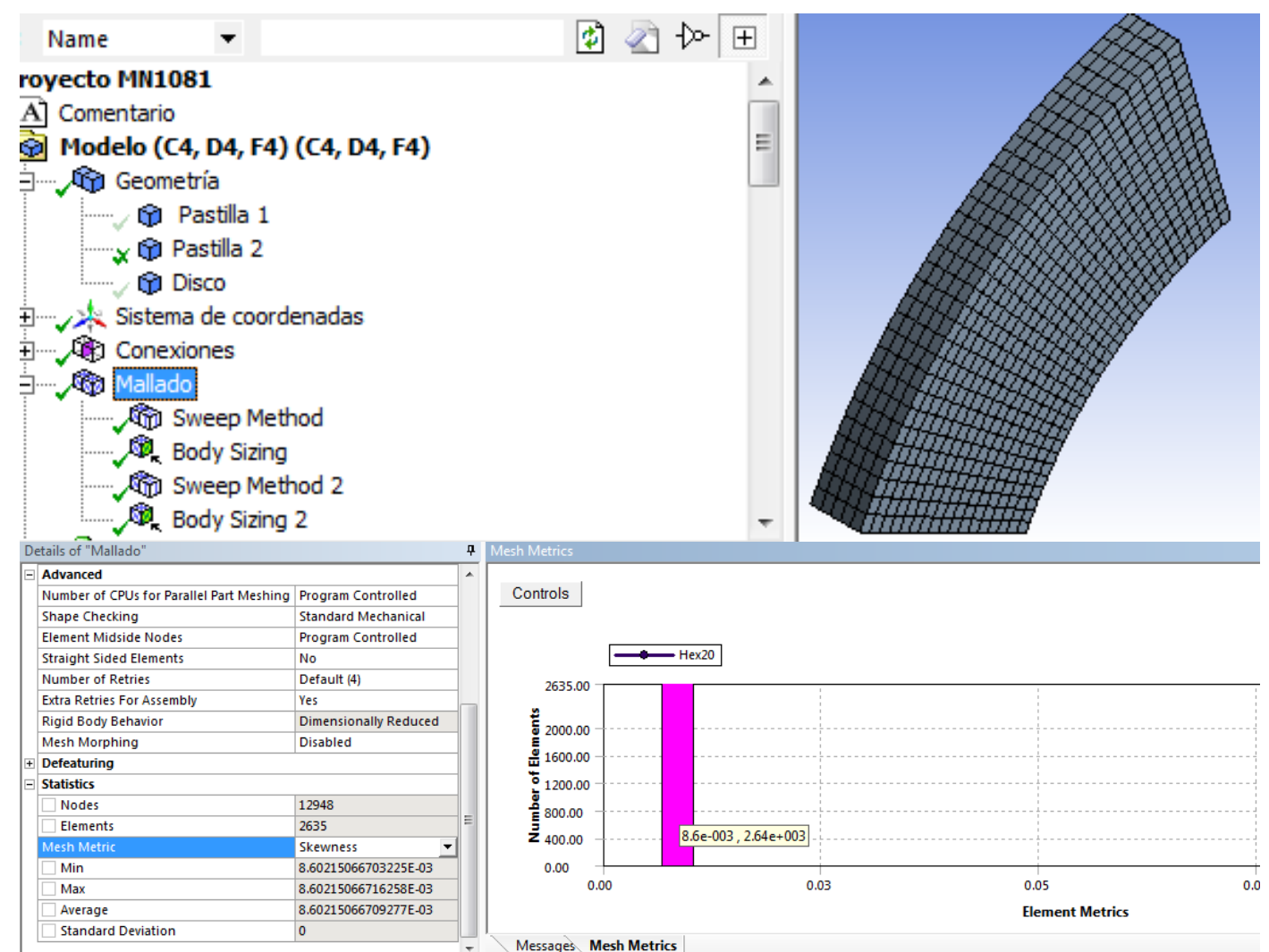

Figura 6. Configuración general de la malla para la pastilla de freno analizado con calidad de oblicuidad

En la Figura 7 se analiza con el criterio de calidad de ortogonalidad y que debe estar en un parámetro de $0.95-1$ como excelente y de $0.70-0.95$ como muy bueno (Díaz, 2000). 


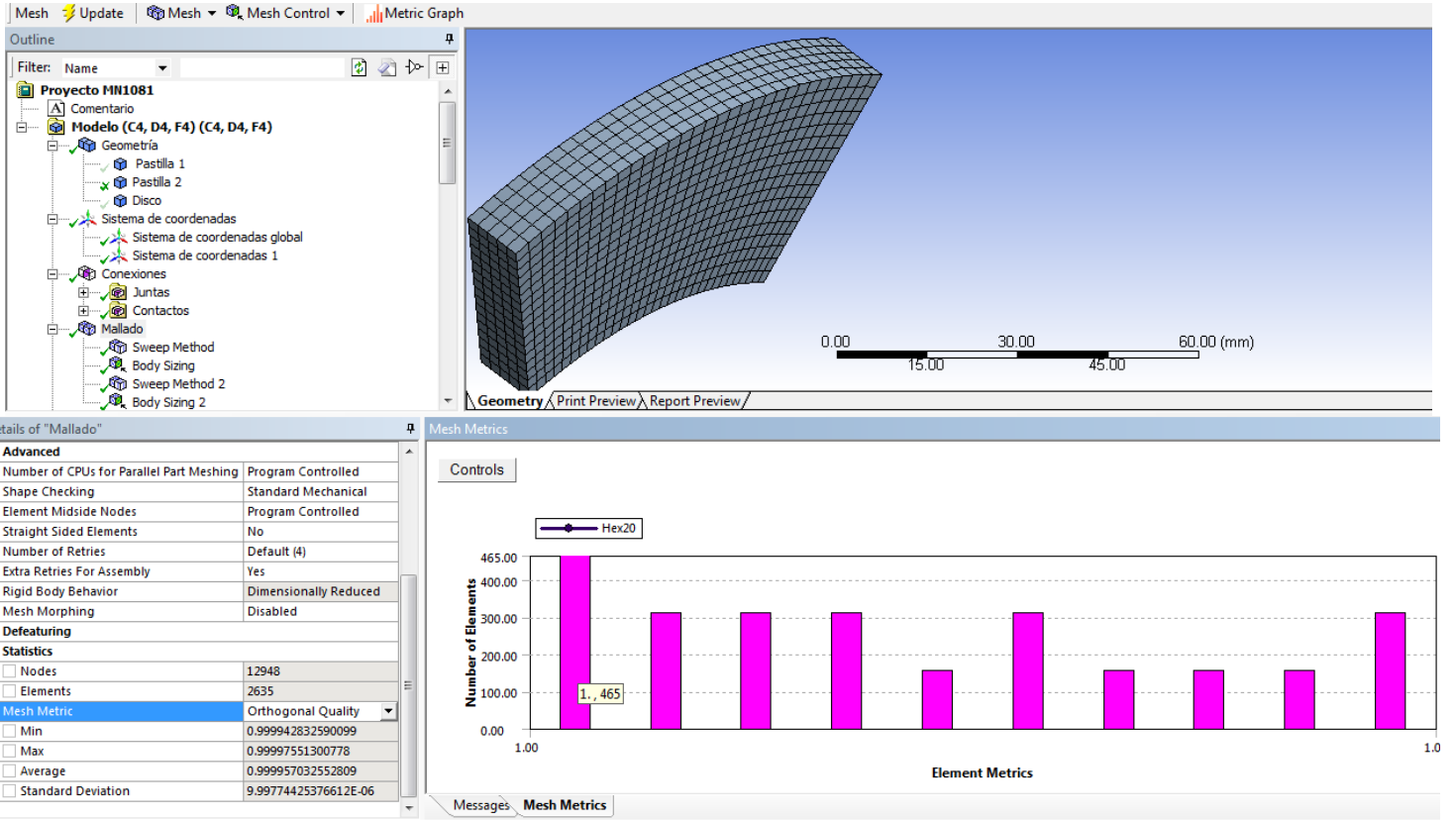

Figura 7. Configuración general de la malla para la pastilla de freno analizado con calidad de ortogonalidad.

\subsection{Aplicación de cargas y soportes}

La aplicación de cargas y soportes dependen de las especificaciones y requerimientos como parámetros de borde del proyecto, se utiliza el sistema multifísico del software colocando las condiciones de trabajo, como se muestra en la Figura 8.

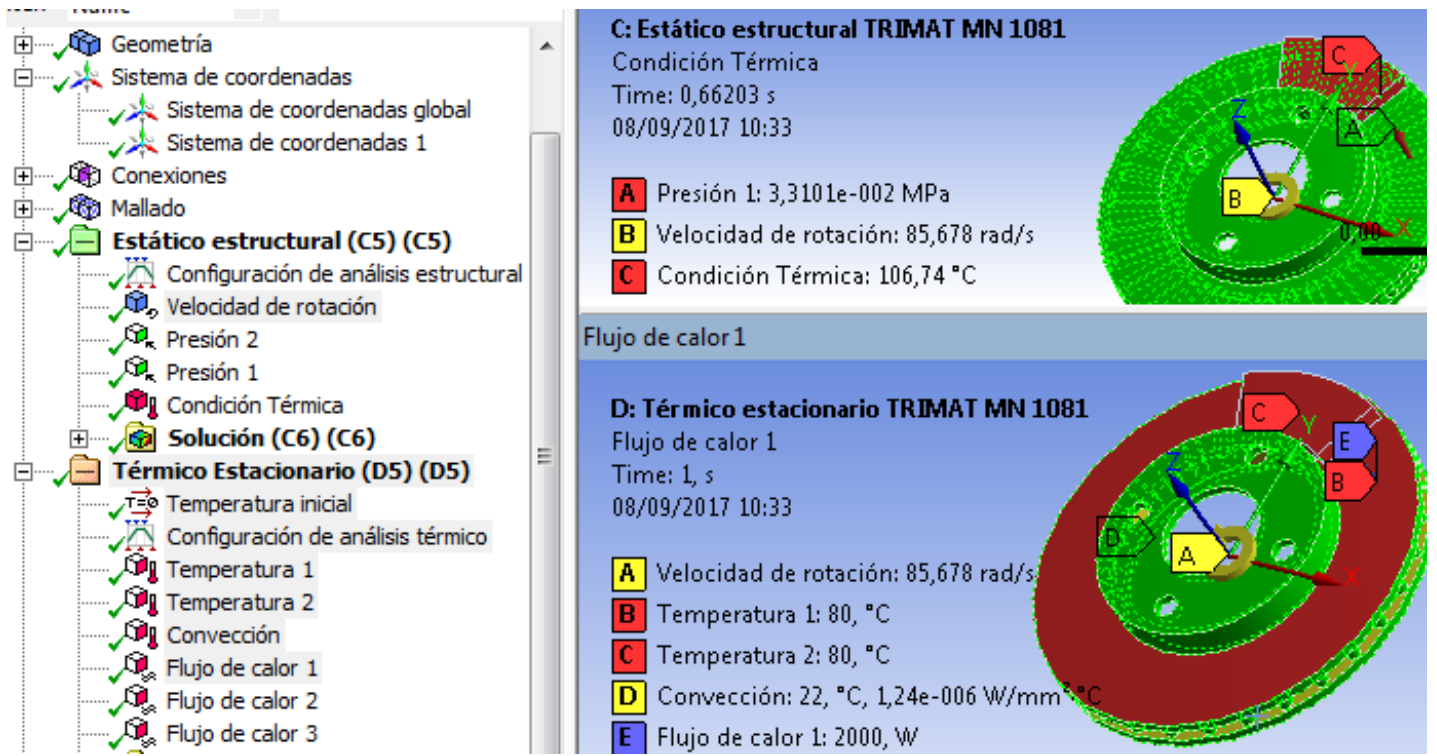

Figura 8. Colocación de los parámetros de borde

En la Figura 9 se muestra el sistema de coordenadas, las conexiones y contactos establecidos entre el disco y las pastillas de freno. 


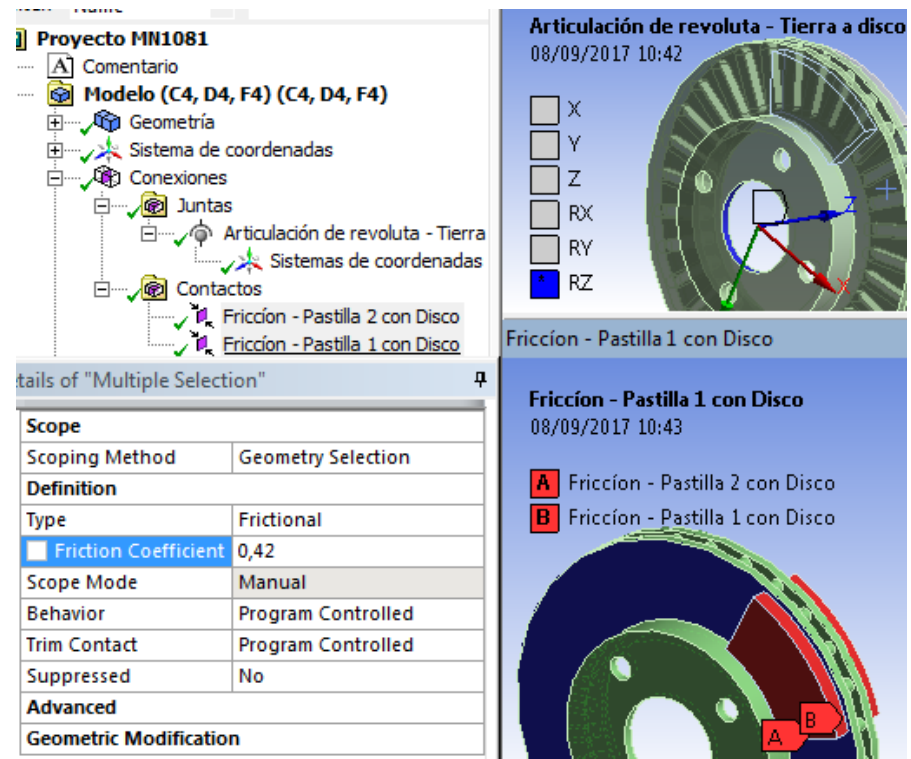

Figura 9. Coordenadas, conexiones y contactos

\subsection{Generación de resultados}

En la Figura 10 se observa la convergencia de la carga generada por la presión de la pastilla al disco para un desplazamiento de $5 \mathrm{~mm}$ y una de velocidad $90 \mathrm{Km} / \mathrm{h}$ en 121 iteraciones (Venegas, 2012).

En las primeras iteraciones fluctúa con una mayor amplitud, a medida que las iteraciones se incrementan su amplitud se reduce manteniéndose de una manera uniforme y esta estabilidad es tomada como la convergencia (Venegas, 2012).

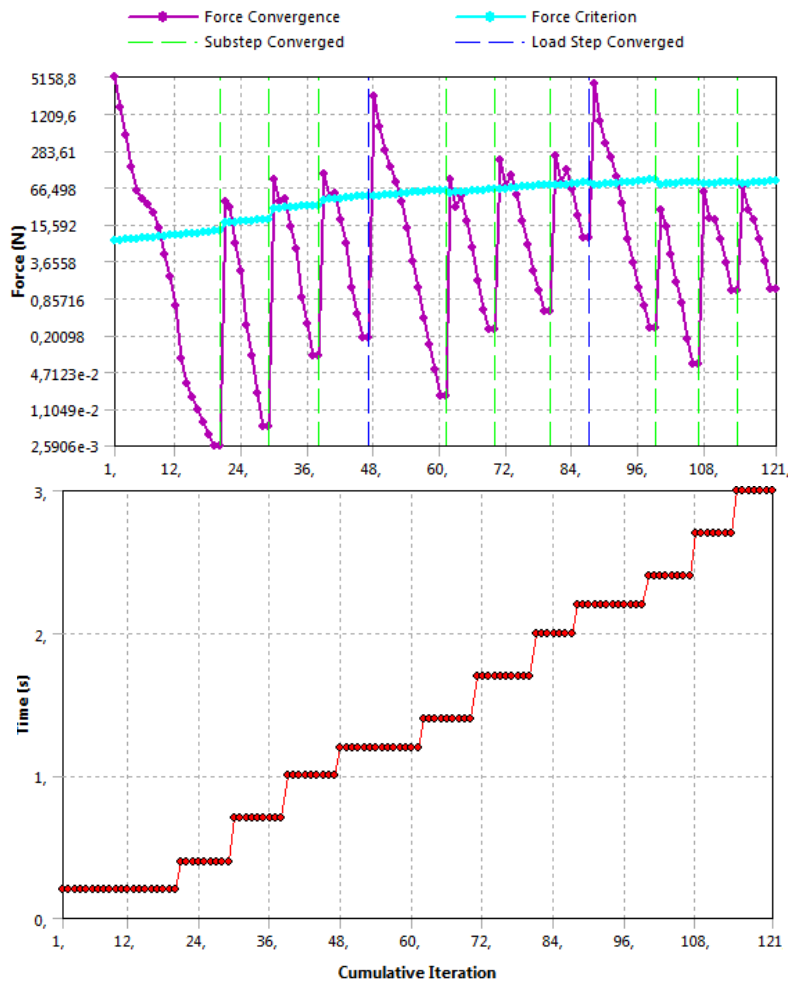

Figura 10. Convergencia de la carga generada 
En la Figura 11 se muestran los resultados del esfuerzo equivalente de Von-Mises, en la Figura 12 la penetración sobre las pastillas en el proceso de frenado y en la Figura 13 la deformación y diagrama esfuerzo versus deformación de las pastillas.

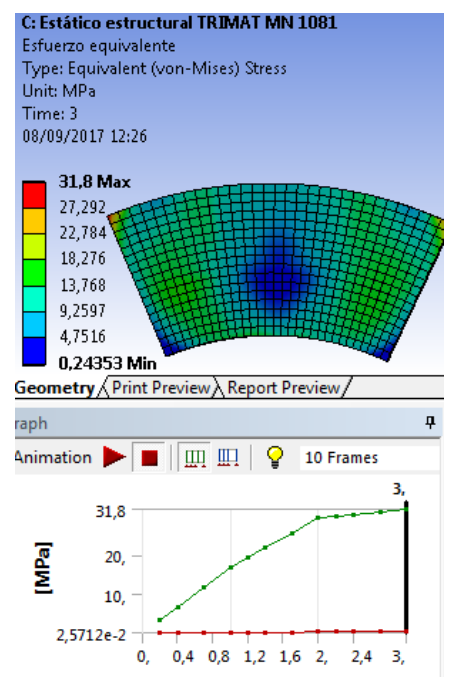

[s]

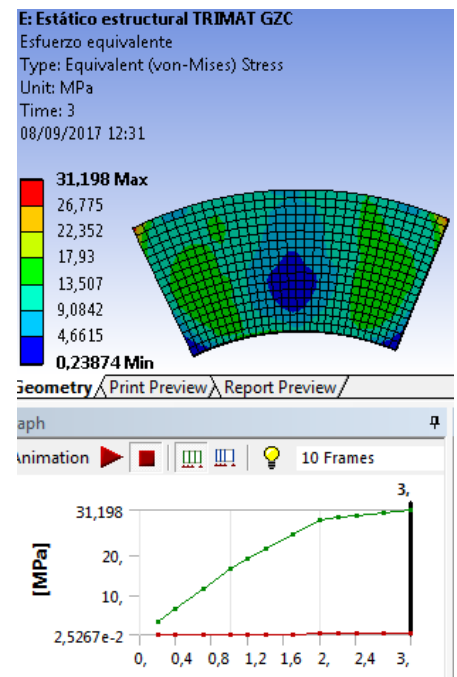

[s]

Figura 11. Resultados de esfuerzos equivalentes
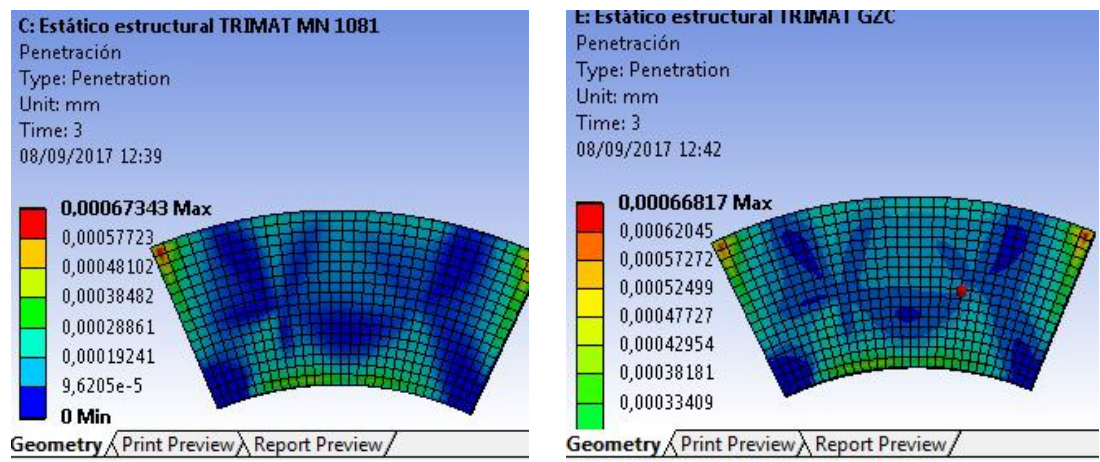

Figura 12. Resultados de penetración en las pastillas
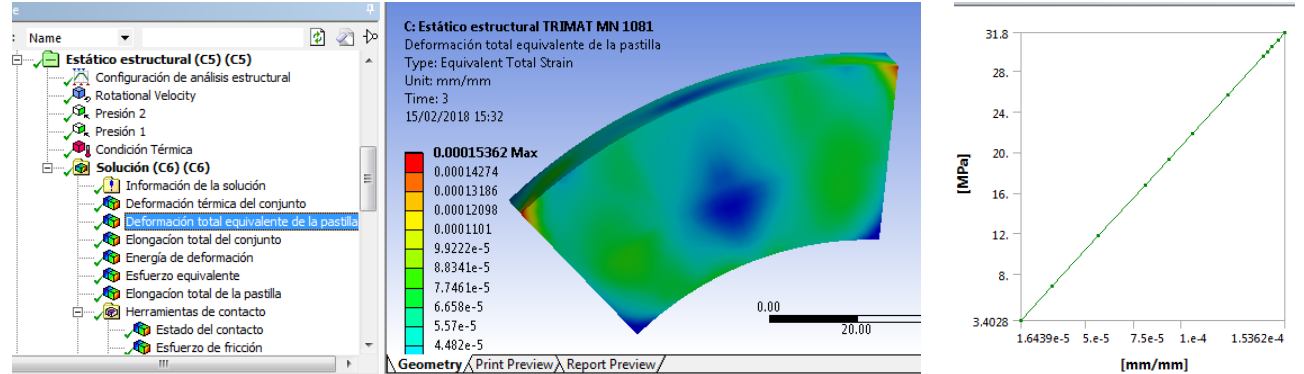

Figura 13. Resultados de deformación y diagrama esfuerzo versus deformación de las pastillas

\section{Resultados y discusión}

En la Figura 10 se observa la convergencia de la fuerza provocada por el contacto entre el disco y la pastilla, dando un calor generado por fricción. Como la amplitud en las iteraciones llega a ser uniforme, esta estabilidad da como resultado la convergencia.

En la Figura 14 se muestra el flujo de calor de las pastillas, mediante un incremento lineal en ambos casos, lo cual implica que se produce un aumento de temperatura uniforme para los dos materiales analizados. 


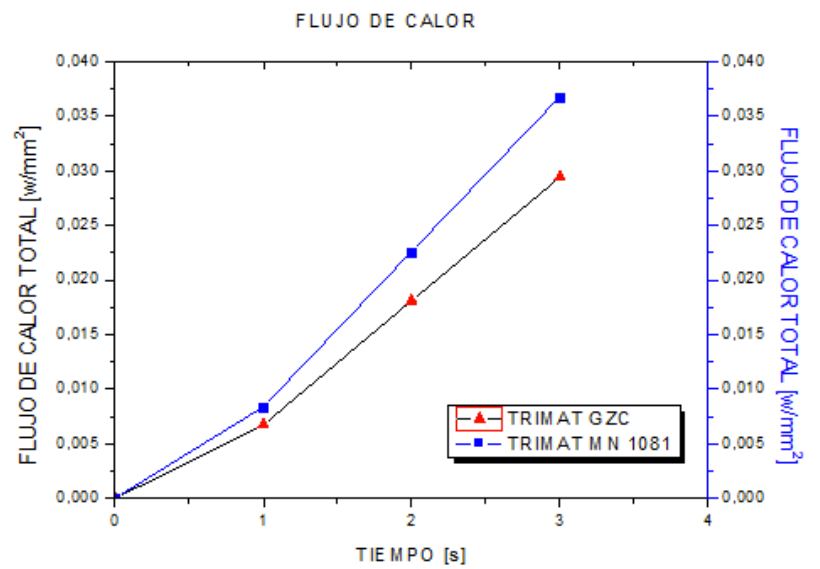

Figura 14. Flujo de Calor

Se verifica que el esfuerzo equivalente para los dos tipos de pastillas no supera al especificado por el fabricante en la Figura 15. Para el Trimat GZC es de 148 MPa y para el Trimat MN1081 es de $62 \mathrm{MPa}$ (TRIMAT friction, Trimat friction solutions, 2012), indicando que no habrá falla del material.
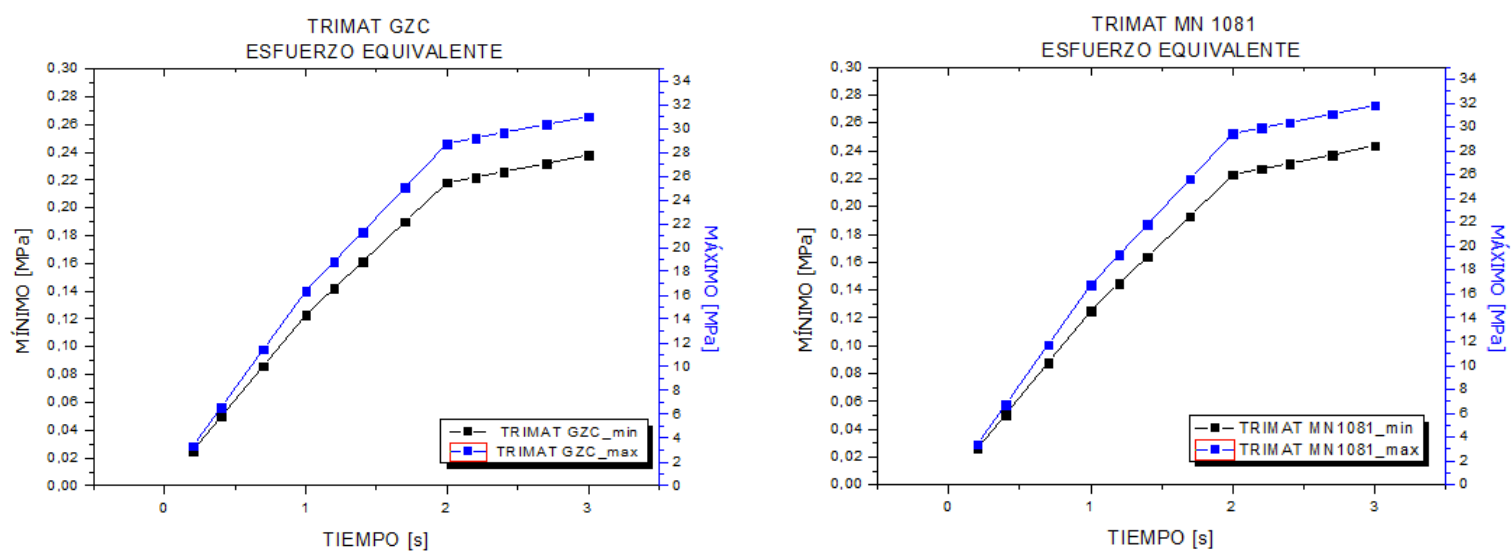

Figura 15. Esfuerzo equivalente

En la Figura 16 se grafica el comportamiento del esfuerzo de fricción dado en las pastillas y que se puede comprobar con el de cizalladura mostrado en el data sheet de cada material. Para el Trimat GZC es de $25 \mathrm{MPa}$ y para el Trimat MN1081 es de $10 \mathrm{MPa}$ (TRIMAT friction, Trimat friction solutions, 2012).
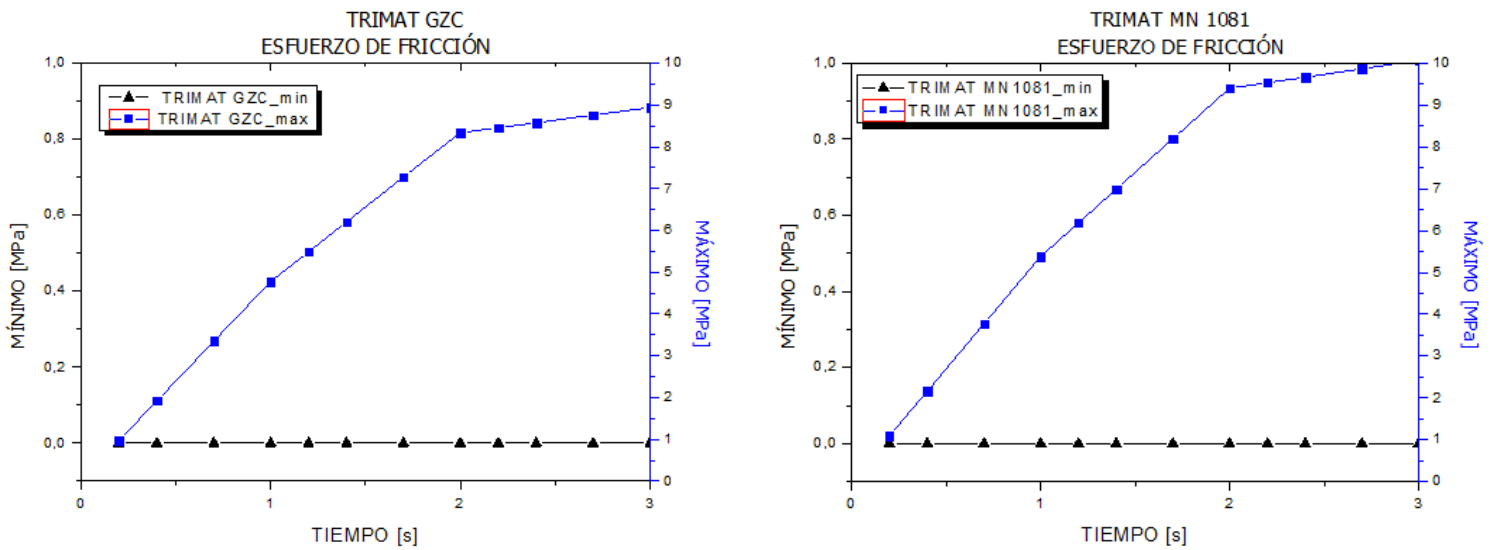

Figura 16. Esfuerzo de fricción 
Para determinar el desgaste de la superficie de las pastillas se utilizan los resultados obtenidos de la penetración del disco en la pastilla por cada frenada mostrada en la Figura 17 y se tiene como máximo $6,7343 \times 10^{-4} \mathrm{~mm}$.
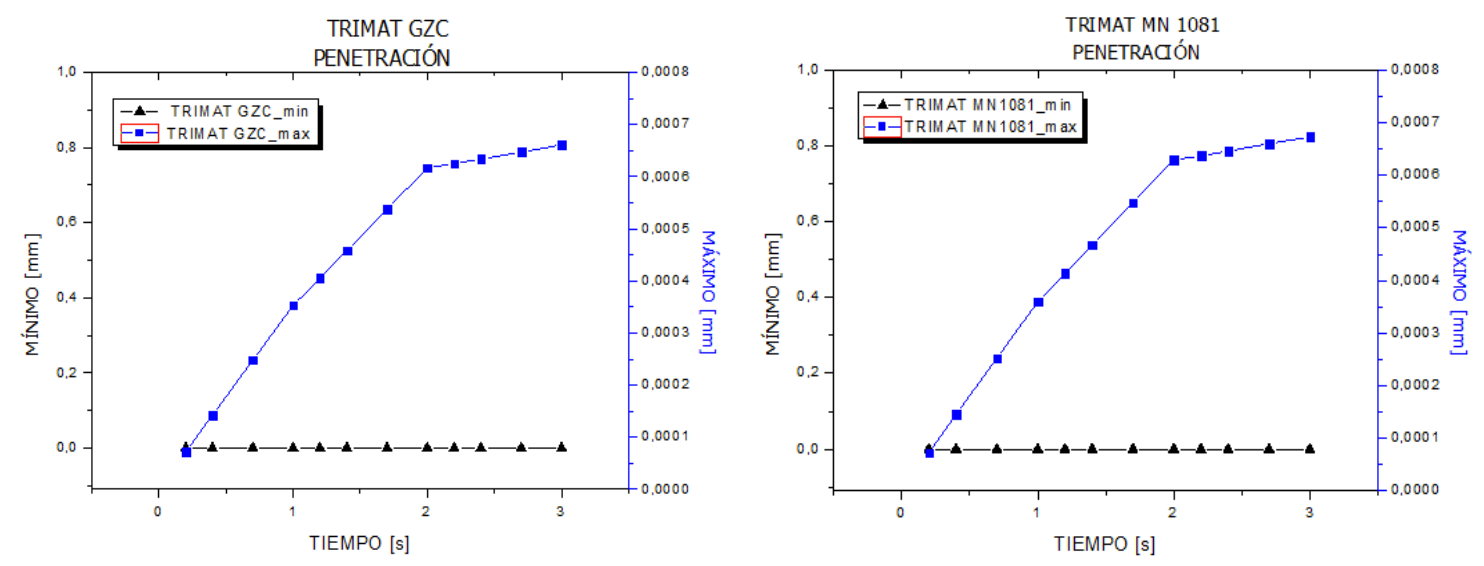

Figura 17. Penetración

Se analiza el comportamiento de la deformación de las pastillas al momento de la aplicación de la carga y proceso de frenado dando resultados en decimas de milímetro, como se observa en la Figura 18.
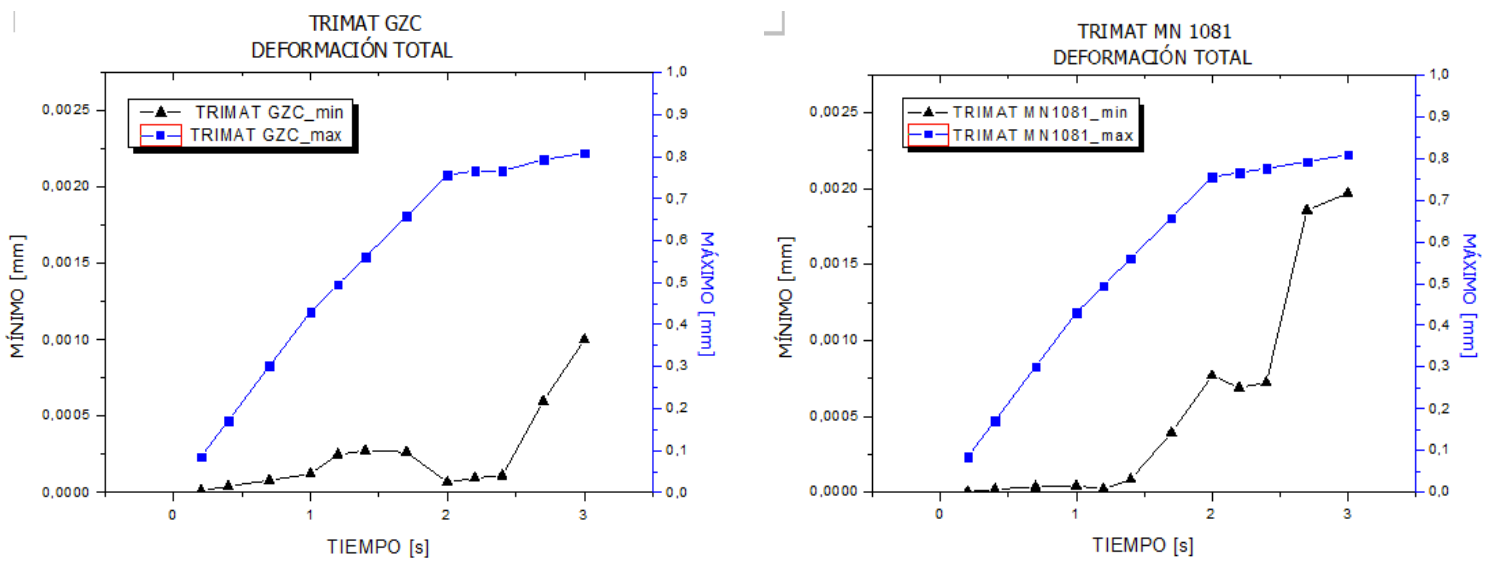

Figura 18. Deformación total

En la Figura 19 se pueden apreciar las curvas de energía de deformación producidas por el sistema de frenado, esta se genera debido a que los frenos trabajan por rozamiento entre una parte móvil que está montada en la estructura de las ruedas y otra parte fija en la estructura del auto.

Al accionar el sistema de frenado, la parte fija se aprieta a la parte móvil y por fricción se consigue desacelerar el vehículo. Esta fricción emite calor y absorbe la energía de inercia.

En el análisis realizado durante un tiempo de 3 segundos se puede apreciar que se genera una cantidad de energía con un valor de $5000 \mathrm{~mJ}$, lo cual corresponde a $1.67 \mathrm{~W}$ por cada frenada.

Los sistemas modernos de frenado están diseñados de tal manera que permiten aprovechar la energía generada mientras se frena, estos sistemas se conocen como frenos regenerativos los cuales permiten reducir la velocidad de un vehículo y transformar parte de su energía cinética en energía eléctrica. 

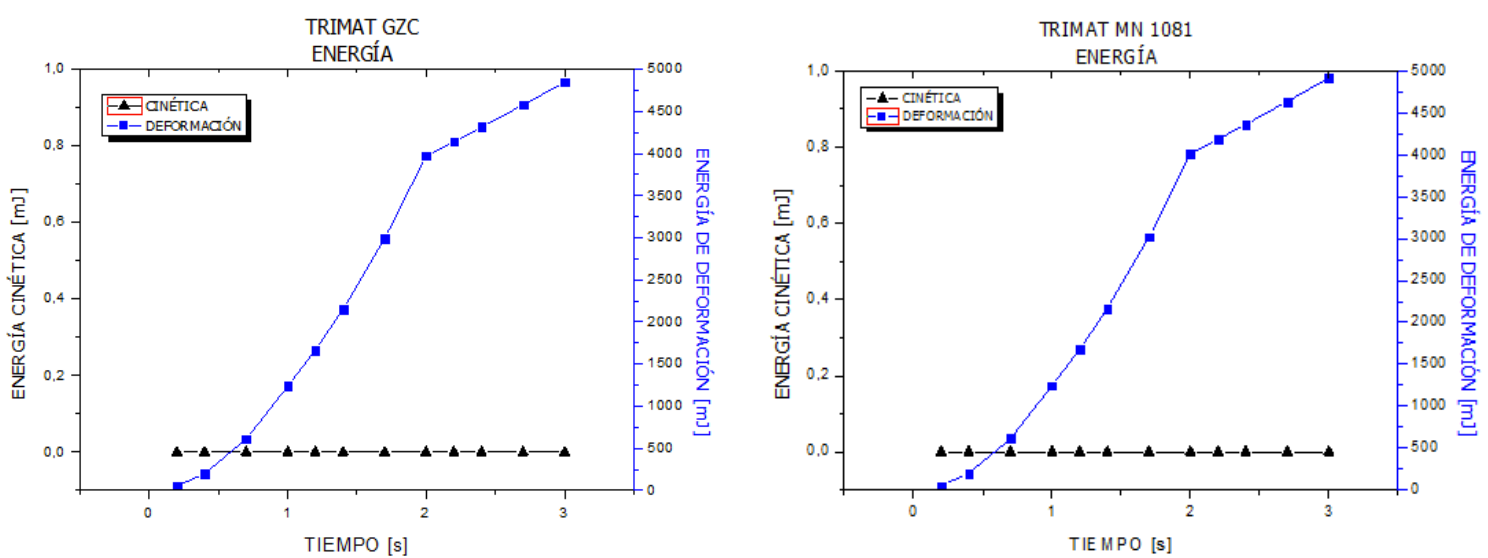

Figura 19. Energía cinética y de deformación.

\section{Conclusiones y recomendaciones}

El análisis por el método de elementos finitos en la interacción de herramientas computacionales de ingeniería, ayuda a mejorar la conceptualización de especificaciones, requerimientos y trabajar con los parámetros de borde definidos y así obtener resultados óptimos para la aplicación física termomecánica del comportamiento de las pastillas de freno.

Para obtener una solución y convergencia de resultados sin un gran coste computacional y tiempo, es primordial realizar un mallado de calidad bajo criterios y herramientas que brinda el software; el utilizado en este trabajo son la oblicuidad y ortogonalidad para un mallado estructurado.

Con este tipo de análisis y el uso del software se puede obtener una gran cantidad de resultados físicos respecto al comportamiento del contacto entre las pastillas de freno y el disco como es el de transformar la energía mecánica por fricción en térmica y que se la puede convertir en eléctrica.

En un trabajo posterior se puede realizar el análisis del sistema de freno con materiales alternativos como la cascara de maíz y palma para comparar los resultados del desgaste provocado por la penetración del disco a la pastilla y compararlo con los resultados mostrados en la Figura 17 para materiales tradicionales.

\section{Bibliografía}

ANSYS. (2012). Ansys advantage-magazine. Recuperado el 16 de agosto de 2017, de http://www.ansys.com/-/media/Ansys/corporate/resourcelibrary/article/AA-V6-I2Full-Version.pdf

ANSYS. (2017). Ansys advantage-magazine. Recuperado el 16 de agosto de 2017, de http://www.ansys.com/-/media/Ansys/corporate/resourcelibrary/article/AA-V6-I2Full-Version.pdf

Benito Muñoz J. J., Álvarez Cabal R., Ureña Prieto F., Salete Casino E., Aranda Ortega E. (2014). Introducción al Método de los Elementos Finitos. Madrid: UNED.

Brown, T., Rothbart, H. (2006). Mechanical Design Handbook. New York: McGraw Hill professional.

Budynas, R., Keith, N. (2011). Diseño de Ingeniería mecánica de Shigley. México: McGraw Hill.

Chand, S., Khurmi, R., Gupta, J. (2005). Machine Desing. New delhi: 14.

Cornejo C. (2010). Sistemas dinámicos con fricción expresado en ecuaciones hamiltonianas controladas por puerto. Ciudad de México: Tesis Doctoral Universidad Nacional Autónoma de México. 
Díaz, A. (2000). Métodos de mallado y algoritmos adaptativos en dos y tres dimensiones para la resolución de problemas electromagnéticos cerrados mediante el método de los elementos finitos. Valencia: Publicaciones de la Universidad de Valencia.

Falasca, G. (2016). Manual técnico pastillas freno. Academia. Recuperado el 16 de agosto de 2017, de http://www.academia.edu/7457719/Manual_tecnico_pastillas_freno

Feng Liu, Xiankuan Qi and Mingqing Sun. (2008). Automation Structural Analysis Based. Informatics and Management Science V, 208, 776. ISSN 1876-1100.

Forsberg, K., Mooz, H., Cotterman, H. (2005). Visualizing Project Management (Modelo en $V)$. Nueva York, NY: John Wiley and Sons.

García A., Castillo F. (2007). CIM: El computador en la automatización de la producción. Castilla: Ediciones de la Universidad de Castilla. ISBN: 10 84-8427-444-6.

ISO21994. (2007). Passenger cars - Stopping distance at straigth-line braking with ABS. Switzerland: ISO.

ISO6310. (2009). Road vehicles - Brake linings - compressive strain test methods. Switzerland: ISO 2009.

Maher Bouazizi; Tarek Lazghab; Mohamed Soula. (2013). FEA of In-Plane Shear Stresses of a Preloaded Sandwich Plate with a Viscoelastic Core: Application to the Disk Brake System. Design and Modeling, 652. ISSN 2195-4356

Moaveni, S. (2008). Finite Element Analysis Theory and application with ANSYS. EE.UU: Pretice Hall.

Naula I., Albuja, G.; Carrillo, R; Izurieta, C. (2016). Modelación y simulación numérica de la Ecuacion de Richards para problemas de infiltración (Vol. 7). Ecuador. Revista Enfoque UTE. 46-58.

Norton, R. (2010). Diseño de maquinaria. México: Mc Graw Hill.

Robert, H. (2002). The mechatronics handbook. Texas: CRS Press.

TRIMAT MN1081. (2012). Trimat friction solutions. Recuperado el 21 de Agosto de 2017 de http://www.trimat.co.uk/pdf/spec-mn1081.pdf

TRIMAT GZC. (2012). Trimat friction solutions. Recuperado el 21 de agosto de 2017 de http://www.trimat.co.uk/pdf/spec-gzc.pdf

Venegas, W. (2012). Análisis por el método de elementos finitos de un disco de freno con sistema mordaza. Trabajos de Investigación. Unidad de posgrados, 173. ISBN: 978-9942-14-000.5

Vinueza A., Gutierrez N. (2017). Análisis por el método de elementos finitos del comportamiento de las pastillas de freno de un sistema ABS discretizando el elemento continuo utilizando software CAE. INCISCOS 2017 (Próximo a publicarse). Quito, Ecuador.

ZhengXin Zhang; FangLin Huang. (2013). Dynamics Analysis of the MRF. Proceedings of the 2nd International Conference on Green and Networks 2012 (GCN 2012), 226, 744. Chongqing, China. ISSN 1876-1119. 